\title{
From Atoms To Functional Nanomaterials; Structural Modifications As Observed Using Aberration-Corrected STEM
}

\author{
Sergio I. Sanchez ${ }^{1}$, Lawrence F. Allard ${ }^{2}$, Melanie T. Schaal ${ }^{1}$, Steven A. Bradley ${ }^{1}$, Gregory J. Gajda ${ }^{1}$ \\ 1. UOP LLC, a Honeywell Company, Des Plaines IL, USA \\ 2. Oak Ridge National Laboratory, Oak Ridge TN, USA
}

For over a decade aberration-corrected scanning transmission electron microscopy (AC-STEM) has proven essential in the study of nanomaterials. Nanomaterials are essentially assemblies of atoms arranged in such a way that they can serve a myriad of applications. Considering their small size, it is not surprising that subtle changes in the atomic structure can significantly impact functionality. In such systems AC-STEM is indispensable, and unrivalled, being able to probe structural modifications with atomistic detail. The current work aims to track functional nanostructure formation starting from dispersed atomic states; this is followed by case studies documenting how modified atomic structure can impact functionality.

The first example examines supported Pt systems as they can serve a variety of functions in science and industry (optics, photovoltaics, catalysis, etc.). $\mathrm{Pt} / \gamma-\mathrm{Al}_{2} \mathrm{O}_{3}$, used in the production of transportation fuels, is a widely studied in catalysis research. In a previous work [1], $\mathrm{H}_{2}$ gas was used to produce $\mathrm{Pt}$ nanoparticles from dispersed atoms. In that study the fraction of $\mathrm{Pt}$ in the form of atoms [to that in the form of clusters] was used to monitor completeness of reduction. We extend these findings to a bimetallic Pt-Re system and examine the impact of changing the gas stream used for reduction to Net gas (a mixture of $\mathrm{H}_{2}$ and light hydrocarbon gases such as $\mathrm{CH}_{4}, \mathrm{C}_{2} \mathrm{H}_{6}, \mathrm{CO}$, etc.). A cost-saving technique practiced by some refiners utilizes Net gas instead of high-purity $\mathrm{H}_{2}$. The exact impact of Net gas reduction on reforming catalysts is not known although a sometimes observed problem is performance loss [2]. When we employed reduction via Net gas on a commercial catalyst, the most clear difference between the reduction methods found was the modification of the particle size (Figure 1); increase in size was observed with Net gas reduction (Figure 1b). On average, the findings showed modifications to nanoparticle size (Figure 2), changes to nanoparticle composition, and differences in single atom to cluster ratio (Figure 1). All these factors collectively would result in a less selective catalyst.

The second component of this work examines metal sulfides which are used as lubricants, as electrocatalysts, and as catalysts in oil and gas refining. We monitor the growth of $\mathrm{WS}_{2}$ nanostructures as they form from $\mathrm{WO}_{\mathrm{x}}$ atomic sub-nanometer clusters (sulfided in $\mathrm{H}_{2} \mathrm{~S} / \mathrm{H}_{2}$ (g) stream) to well-ordered, graphitic-like, sheets of $\mathrm{WS}_{2}$. In the refining industry this $\mathrm{WS}_{2}$ phase is coupled with promoter species such as Ni (or Co) to improve activity. Figure 3a is an AC-STEM image of a W-only sample shown assuming a triangular morphology. Conversely, Figure $3 \mathrm{~b}$ shows the Ni-promoted $\mathrm{WS}_{2}$ nanostructure where edge sites have become less faceted, consistent with other literature reports [2-4]. It has been proposed that the edge site modification stems from incorporation of the promoter atoms into the edge sites. This observation is consistent with EELS work done by others [5] and serves as indirect confirmation of the promoter species terminating the edge sites.

To quantitatively understand the change in dispersion the perimeter (P) and area (A) for a series of individual $\mathrm{WS}_{2}$ sheets was measured. Presumably, if the edge sites are the active component of these nanomaterials then it follows that an increased dispersion (a higher P/A ratio) would be beneficial to 
performance, this is observed in Figure 4 for the Ni-promoted sample. Testing results for these two catalysts showed clear improvements in yield and activity with $\mathrm{Ni}$ addition.

Lastly, an attempt is made to understand whether a W-only sample with comparable P/A ratios to the W$\mathrm{Ni}$ system could achieve the same performance. A successful attempt was made when preformed $\mathrm{WS}_{2}$ sheets received an oxidation treatment produced nanostructures with similar edge site modification as the Ni-promoted samples. The findings are important as they suggest an alternative method to introduce less faceted edge structures.
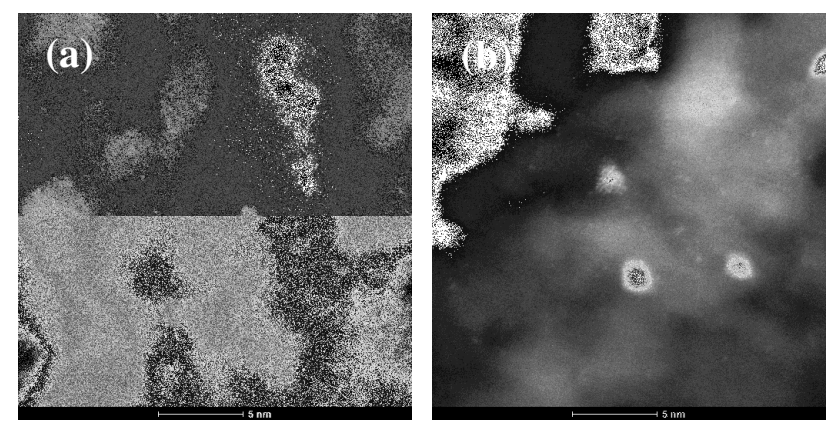

Figure 1. Atomic resolution STEM images of Pt-Re nanoparticles formed by (a) $\mathrm{H}_{2}$ and (b) Net gas reduction
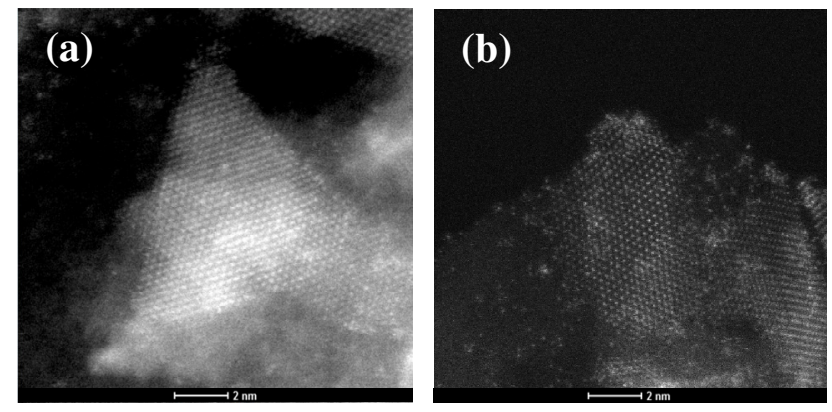

Figure 3. Atomic resolution STEM images of (a) unpromoted and (b) promoted $\mathrm{WS}_{2}$ sheets

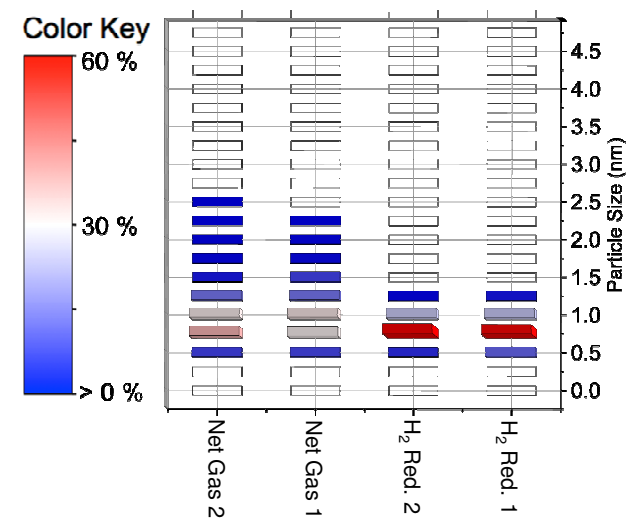

Figure 2. Size distribution histograms comparing two samples reduced in $\mathrm{H}_{2}$ gas compared to two different samples reduced via Net gas.

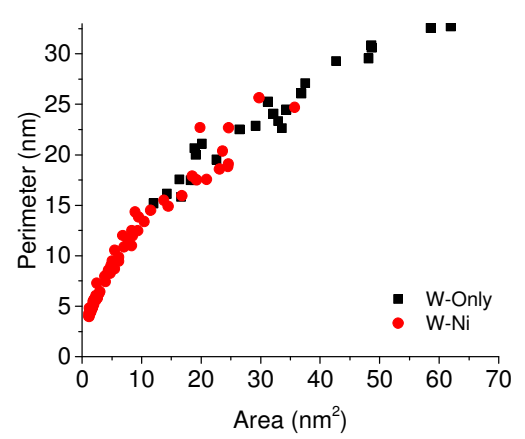

Figure 4. Relationship of perimeter to area for a series of promoted \& unpromoted $\mathrm{WS}_{2}$ sheets

[1] SI Sanchez et al., Micros and Microanal. 20 (2014) p. 1656

[2] PK Doolin, et al. "Catalytic Naphtha Reforming." $2^{\text {nd }}$ Ed. GJ Antos and AM Aitani (Marcel Dekker, Inc New York, NY) p.444-445

[2] M Girleanu et al., ChemCatChem 6 (2014), p. 1594.

[3] J Kibsgaard et al., Journal of Catalysis 272 (2010), p. 195.

[4] LP Hansen et al., Angewandte Chemie International Edition 50 (2011), p. 1.

[5] Y Zhu et al., Angewandte Chemie International Edition 53 (2014), p. 10723.

[6] A portion of this research is supported by the U.S. Department of Energy, Office of Science User Facility operated at Oak Ridge National Laboratory 